Vomiting almost invariably occurs at the outset of scarlet fever; very rarely in the other acute exanthems, and does not recur, except in very grave cases. In affections of the brain, particularly after injuries, as by blows upon the head or concussion of this organ from any cause, vomiting is a constant symptom. It is an early concomitant of cholera infantum. It must also be remembered that vomiting is frequently the first sign that the breast milk is disagreeing with the child, because of pregnancy, or other condition improper for the continuance of nursing. The diagnosis is here made by the constant recurrence of this symptom without the advent of others, unless it be diarrhoea or the evidences of want of nourishment. Vomiting often ensues immediately when a child is nursed by an excited or exhausted mother. Here, too, coma or convulsions frequently follow.

Vomiting will occur in many infants that are deprived of the breast milk, or where an improper form of food is employed. With many children, it be comes a matter of great difficulty to find food that will agree with, or even be tolerated by the stomach.

The breath of an infant shoull be odorless. It becomes acid from an excess of acid in the food. All odors of the breath should attract attention, especially that of foetor. This demands a careful examination for ulceration of the cheeks, throat, nose, etc. Occasionaliy it has been the earliest notice of the presence of diphtheritic throat.

I'he evacuations from the bowels should always be carefully inspected. Rarely can so much be learned by a description as by an inspection. Hence the nurse should reserve each diaper as removed, or several separately when convenient, and without the ad mixture of anything which would change the appearance of its contents. Sometimes a number of evacuations, alarming by their number, when combined will only amount to what should ordinarily be passed at once. Errors in diet are readily detected by the presence of particles of undigested food, seeds, skins, curd, and a variety of foreign bodies. Thus may be shown the presence of parasites, acidity, blood, mucus, the want of bile, and other disordered conditions. Blood mixed with mucus, febrile symptoms being present, would indicate dysentery. Pure blood would demand an examination for an injury, a foreign body, a polyp, and would be a symptom of purpura hæmorrhagica. Difficult passages, with blood or small discharges of blood, and the absence of the ordinary matters, would indicate constriction of the bowels, intussusception, etc. Dark green discharges or black tarry evacuations continuing for days after the birth of the child, show that the meconium has not been completely expelled.

Cold causes the passage of slimy mucus. The absence of one or more evacuations each day is important to notice, lest when other symptoms do not present a habit should thus be contracted. Colic is thus produced, inflammation of the bowels, or the straining to cause an evacuation may end in prolapsus ani, hernia, or other injury. In obstinate constipation the use of opiates may be suspected, and a most rigid incuiry should be made.

The urine if yassed with pain or suppressed, de- mands an examination of the genitals to detect the presence of a stone in the urethra, an agglutination of the orifice of the prepuce, etc. The urine becoming scanty or disappearing after an acute attack, as scarlet fever, indicates disease of the kidney and dropsy.

A very frequent trouble is constantly overlooked, or regarded in a wrong light. This is incontinence of urine. Much injustice is caused by a want of knowledge as to the nature of this affection. Parents, supposing it due to natural uncleanliness, or to laziness, endeavor to correct it by severe punishment. Again, by many it is regarded as utterly incurablea belief which is, unfortunately, shared by members of our profession and thus neglected, children are suffered to grow to years of maturity the victims of a disgusting malady. Even in these cases, in many instances, it has proved amenable to treatment.

Night terrors, that peculiar condition which causes even older children to spring screaming from the bed at night, or to scream and cover their heads with the clothing, should not be disregarded or treated as willfulness. It is a symptom imperatively demanding attention, and one which if its causes are not removed may eventuate in idiocy, or later in insanity.

Finally, a careful grouping of symptoms, the elimination of causes of errors, a thorough examination of the infant both during repose and when aroused; and, in the event of difficulty in making the diagnosis, with the clothing entirely removed will rarely fail to enable the practiced and observant physician to arrive at a positive conclusion as to the nature and cause of the affection, and give him a clue to the proper line of treatment.

\section{IS ALCOHOL ESSENTIALLY A STIMULANT OR A} PARALYZANT?

BY A. B. PALMER, M.D., LL.D., PROF. OF PATHOLOGY AND PRAC'TICE OF MEDICINE, IN THE COLLEGE OF MEDICINE AND SURGERY, UNIVERSITY OF MICHIGAN.

For many years past, from my own observations and experience, I have been convinced and have taught, that alcoholic drinks should not be spoken of as stimulants - as though their leading effect was the increase of power or activity in the system. That in certain conditions of disease, of shocks from injuries or suffering, and in some persons habituated to their use, they increase action temporarily, I have admitted and still admit; but that their effect in the physiological condition is to increase action, at least to any useful extent, even temporarily, in whatever quantity used, I have for a very long time doubted, and for several years past have very confidently denied. In the particular function where the most positive and ready test can be appliedthat of muscular power-experiments have always shown that no quantity of alcohol, small or great, can increase that power in ordinary heaithy conditions. One lifting all he is able cannot be made to lift more by taking alcohol. Experiments in France and elsewhere have invariably shown that when suf- 
ficient alcohol was taken to produce an appreciable effect upon the muscles, their power has been diminished and not increased. It has been proven that this is the case not only with men, but with other animals. The horse, in the races when strength and fleetness are most exactly tested, cannot be made to run faster by any alcoholic dose; but on the contrary is weakened and rendered helpless by it.

These facts have long been known, though the proper conclusions from them have not always been drawn. Men who indulge in alcohol often fancy themselves stronger from its use. When so much weakened by it that they can scarcely stand, they often boast of their strength prompted by a deceptive feeling. When it is taken in smaller quantities, the same delusion is often produced. Those who habitually use alcohol, opium, or even tobacco, feel depressed when deprived of their accustomed narcotic, and are revived by returning to it; but no physiologist thinks of calling tobacco a "stimulant" because of this. All contend that its effect is essentially sedative--a diminisher of action. The writings of the late Dr. Anstie, of London, though he was not an advocate of complete abstinence from alcoholics, have done much to call attention to the precise action of these articles, and oppose the more common but erroneous view of their "stimulating" qualities.

The views of Dr. Richardson, the eminent sanitarian, physiologist and physician of London, on this subjectare well known, but as years ago he followed his physiological views to their practical conclusions, and became an ardent advocate of total abstinence, a practice and a cause at that time, and still with some, unpopular in England, his opinions founded on scientific experiment and careful observations have not exerted the influence among scientific men to which they are entitled. More recently other scientific men, not connected with any special temperance movement, have expressed opinions on this subject which must have much influence in changing professional opinions and expressions, though long established custom of expression, belief or practice is slow to change.

Dr. Samuel Wilkes, of Guy's Hospital and Medical School, and one of the most acute and independent thinkers in the profession, in Fngland and elsewhere, in an article in the Cotemporary Kevew, since published with other articles on the subject in a book, says :

"If most persons analyze their sensations after imbibing any alcoholic drink, they will soon discover that to describe the effect produced upon them by it as stimulating, is a misnomer; and that, consequently, the employment of the expression almost begs the whole question as to its operation and value; for there can be but little doubt that it is owing to this misapplication of the term stimulant to alcohol, with many conveying an idea of strength, that causes it to be so much recommended, and taken with so much satisfaction. If a person is low and a glass of wine produces a pleasurable effect, it is easy to regard it as a stimulant, and as having afforded some proportion of strength."
This, he thinks, is a mistake. He further says : "Its stimulating effects may be regarded as nil compared with those which may be styled its sedativ'e or paralyzing ones. In a word, alcohol for all intents and purposes may be regarded as sedative or narcotic, rather than stimulant." $\mathrm{He}$ classes it with opium, Indian hemp and tobacco. It doubtless may sometimes temporarily soothe a worried, nervous system, but its secondary effects cause more worry, which worry it may again soothe. But by these repetitions the alcoholic habit and all it sad effects of chronic alcoholism follow.

But other means of testing this question of the stimulating or sedative effects of alcohol more precise and measurable, if not more satisfactory, are used. One of the latest series of experiments which has come under my observation was made by Sidney Ringer, M.D., author of an excellent work on materia medica and therapeutics, and professor of the practice of medicine in University College, I.ondon, together with Harrington Sainsbury, M. D., M. R. C. p., published in May number, $\mathbf{1} 88_{3}$, of The Practitioner, a journal of therapeutics and public health, edited by T. Lauder Brunton, M.D., F. R. S., Fellow of the Royal College of Physicians, lecturer on materia medica and therapeutics in St. Bartholomew's Hospital School, etc.

The object of these experiments was to ascertain the strength of the different alcohols, and they were made upon the hearts of frogs. They were conducted in a strictly scientific manner, with the most approved instruments of precision now so frequently in use by original investigators in physiology.

All the alcohols were found to diminish the power and soon to stop the action of the heart; and the experiments were so managed in the light of previous experienceas to have the complete arrest occur in about an hour; and the strength of the different alcohols was determined by the quantity required to produce the effect.

It was found, according to these experiments, that the action of all the alcohols was essentially the same in kind, differing only in degree. All arrested the heart in diastole-that is, stopped it in a state of relaxation or paralysis. None of them increased the power of the heart at any time or in any desree of their action, but diminished it from the first, and until it was arrested.

Of the methylic alcohol $\left(\mathrm{C} \mathrm{H}_{4} \mathrm{O}\right)$, the lightest and most powerful of the series, 205.5 minims were required to stop all action in the given time. Of the ethylic $\left(\mathrm{C}_{2} \mathrm{H}_{6} \mathrm{O}\right)$, the common alcohol, it required Ir 4 minims. Of the next heavier, the propylic $\left(\mathrm{C}_{3} \mathrm{~A}_{8} \mathrm{O}\right), 59.3$ minims were required. Of the butylic $\left(\mathrm{C}_{4} \mathrm{H}_{10} \mathrm{O}\right)$, I 7 minims were required; while of the amylic, the heaviest $\left(\mathrm{C}_{3} \mathrm{H}_{12} \mathrm{O}\right)$, only 6.6 minims were required to stop action.

These articles, properly diluted, were caused to pass through the heart, and were thus applied directly to its tissue, and may not represent the action of the articles correctly in all respects as applied to the general complex human system ; but the experiments showed clearly the essential action of the agents, and demonstrated more positively their paralyzing, and 
the absence of their stimulating effect, than their application to a complex organism, where paralysis of inhibitory or restraining functions may result in increase of certain actions.

These experiments, the authors declare, demonstrate more definitely than any others that have been instituted the essential qualitative similarity of the action of all the alcohols-their sedative effects-and a certain general quantitive relationship of one to another. It is scientifically interesting to the chemist and the physiologist to know that as the complexity of the molecular combination increases, the physiological activity, or poisonous effect, increases.

There are some small quantities of these heavier alcohols in various liquors, and certain combinations of them are said to constitute the fusil oil. The quantity compared with the 50 per cent., more or less, of common alcohol in spirits, is not often sufficient to modify the effect to any appreciable extent, especially as the qualitative effects of all the alcohols are so similar; but so far as they are present they increase the poisonous qualities of the liquors containing them.

The report of Drs. Ringer and Sainsbury closes with the remark, announcing the most important practical fact which these experiments confirm, viz., " that by their direct action on the cardiac tissue these drugs are clearly paralyzant, and that this appears to be the case from the outset, no stage of increased force of contraction preceding" (Practitioner, May', $1883, p .35^{\circ}$ ).

In another part of the report the authors say: "The position alcohol occupies is that of a narcotic, and it is probable that its action is very similar to that of ether. $* *$ The sphygmographic experiments of Parkes and Wollowicz on man showed clearly the accelerating effect [of alcohol], but gave no distinct indication of increased arterial pressure." The arterial pressure is the evidence of the increased force. Increased frequency of pulsation is often the strongest evidence of diminished force or power, a very rapid, fluttering pulse usually occurring in extreme weakness.

All agree that the ultimate effect of any considerable amount of alcohol is depressing and paralyzing, and that in chronic alcoholism all the conditions indicate the failure of power; but yet many, even medical men, at least by their use of language, seem to think that in moderate quantities and as frequently used it is a stimulant, and some perhaps still regard its depressing and fatal effects as the result of overstimulation.

With the facts now presented before us, and others of a similar character so constantly accumulating, it cannot be long before our ideas and our language respecting alcoholic drinks will more nearly conform with the teachings of science.

\section{A CASE OF TYPHLITIS, WITH AUTOPSY,}

BY R. D. BARKER, M.D.

Luella A., American, aged twenty-one years. Was called to see her first at 2 P. M., July 5, 1883 . Learned the following history of the case: Had been for some time of a costive habit-bowels frequently not moving for three or four days. Although not feeling very well, she went on an excursion to Spirit Lake, twenty"miles distant by rail July 4. The forenoon of the day was damp and rainy, and upon arriving at the lake she was too ill to join her companions in the grove, but lay on the sofa in the sitting-room of the hotel most of the day. She complained to her friends of pain "across her," and vomited once or twice during the day.

On returning home in the evening, not having had movement of her bowels for two or three days, and the pain across her continuing, her mother gave her a dose of salts which she vomited. In the evening after her return she attended a dance, but was too ill to stay long; was in pain all night, the night of the 4th; the morning of the fifth, her bowels remaining costive, her mother gave her some senna tea which she vomited. I found her at 2 P. M., the $5^{\text {th }}$ of July lying dressed on a sofa; had been crying, and seemed very low spirited, anxious and dispondent; very little febrile excitement; pulse 92 ; temperature normal ; tongue slightly coated; her menses had appeared two weeks ago, and she was regular in her periods; she complained of severe pain in lower part of abdomen, extending to the pubes ; excessively tender over the region of pain; very little tympanites; urine normal in quantity (so she said), but voided with pain ; bowels not moved for three days. The pain being solow down and so severe, without the grave constitutional disturbance indicative of peritonitis, I diagnosed neuralgia of womb and appendices, and ordered copious vaginal injections of water as warm as could be borne; to apply hot applications over abdomen, and

B Fluid extract gelsemini.............gtt. ix Fluid extract belladonna.............gtt. xvi Simple Elix............... $3 \mathrm{vi}$

M. Sig. Teaspoonful every two hours.

Was called at I I p. m.; medicine had given no relief; lies on the sofa, with knees drawn up, face flushed; pulse I 40, and depressed; abdominal tenderness very marked, patient crying out in agony.

I question mother, and learn that she had not urinated to-day, and that they had misunderstood my orders, and had given her one quite large warm water enema into the rectum, which had produced two free liquid discharges. I pass catheter, but obtain no water; I introduce $1 / 2$ grain suppository of sulph morphia into rectum; I ask for counsel, and Dr. J. Croft is called. We wait half an hour for the effects of the suppository, and then find it necessary to give her by hypodermic injection $1 / 8$ grain sulph. morphia and $1 / 120$ grain atropia. This in the course of an hour gives her much relief, and we leave her with instructions to introduce the suppositories every two hours if necessary to relieve pain, and to continue hot fomentations.

Saw her again at 9 a. m.; now thoroughly under the influence of the morphia, but hardly free from pain; has had nausea since early morning, and has vomited several times, greenish, bilious fluid, containing no focal matter; pulse 150 , and weak; body bathed in cold, clammy sweat; complains of intense 